\title{
Factores determinantes del rendimiento académico de los estudiantes de la Universidad de Atacama
}

\author{
Determinant Factors of Academic Achievement of University of Atacama Students \\ Fatores determinantes do rendimento acadêmico dos estudantes da \\ Universidade de Atacama
}

\section{Planck Barahona $U$.}

Facultad de Ingeniería, Departamento de Industria y Negocios, Universidad de Atacama. Telf.: (56) (52) 206500

Correo electrónico: planck.barahona@uda.cl

\begin{abstract}
RESUMEN
El objetivo de este trabajo es determinar los factores asociados al rendimiento académico, para el año lectivo 2010-2011, de los estudiantes de primer año de las carreras de Trabajo Social, Ingeniería, Derecho y Humanidades de la Universidad de Atacama (UDA), para un total de 258 alumnos. El trabajo lo hemos dividido en dos partes; en la primera, hemos creído conveniente utilizar el modelo de Regresión Múltiple con datos de corte transversal para determinar las variables predictoras del rendimiento académico; en la segunda, hemos considerado igualmente importante estimar las variables que inciden en la probabilidad de mejorar el rendimiento académico del estudiante haciendo uso del Modelo de Regresión Logística. Luego del análisis, los resultados han puesto de manifiesto que las variables género, estudia y trabaja, conformidad con la carrera, notas prueba verbal y matemática, resultaron ser estadísticamente significativas. Es decir, tendrían un efecto positivo sobre el rendimiento académico del estudiante.
\end{abstract}

Palabras clave: rendimiento académico, Universidad de Atacama, modelo de regresión múltiple, modelo de regresión logística.

\begin{abstract}
The aim of this study is to determine the factors related to academic performance for the 2010-2011 academic year, in students of first year of Social Work, Engineering, Law and Humanities of the University of Atacama, for a total of 258 students. This work has been divided into two parts. In the first part, we decided to use the multiple regression model with cross-sectional data to determine the predictors variables of academic performance. In the second stage, we considered equally important to estimate the variables that affect the probability of improving student achievement using Logistic Regression Model. After the analysis, the results have shown that the variables such as: the student's gender, work, compliance towards the major, and the grades in the math test and verbal aptitude test, were statistically significant. In other words, that they might have a positive effect on the student academic achievement.

Key words: academic achievement, University of Atacama, multiple regression model, logistic regression model.
\end{abstract}

\section{RESUMO}

Determinam-se os fatores associados ao desempenho acadêmico para o ano letivo 2010-2011 dos estudantes de primeiro ano de Serviço Social, Engenharia, Direito e Humanidades da Universidade de Atacama (UDA) para um total de 258 estudantes. O trabalho divide-se em duas partes: na primeira, acreditou ser conveniente usar o Modelo de Regressão Múltipla com dados de corte transversal para determinar as variáveis previstas no desempenho acadêmico; na segunda, considerou-se igualmente importante estimar as variáveis que afetam a probabilidade de melhorar o desempenho acadêmico do estudante, utilizando-se o Modelo de Regressão Logística. Após a análise, resultados mostraram que as variáveis de gênero, estudo e trabalho, conformidade com a carreira, notas da prova verbal e matemática, foram consideradas estatisticamente significativas. Ou seja, têm um efeito positivo sobre o desempenho acadêmico do estudante.

Palavras chave: desempenho acadêmico, Universidade de Atacama, modelo de regressão múltipla, modelo de regressão logística. 


\section{INTRODUCCIÓN}

En los últimos años han aumentado las aportaciones teóricas y los trabajos empíricos con el objetivo de determinar las causas del rendimiento académico de los estudiantes de la educación superior. Existe un consenso en que los factores asociados al desempeño académico pueden tener su origen en dos grandes ámbitos: en los determinantes personales y en los determinantes sociales. En el primero de ellos se incluye: la inteligencia, las aptitudes, la asistencia a clases, el género, la nota de acceso a la universidad. En el segundo, el entorno familiar, el contexto socioeconómico, las variables demográficas (sexo, edad, estado civil) y la escolaridad de los padres. Se cree que el conocimiento de estos factores asociados al rendimiento podría permitir a las autoridades universitarias diseñar políticas tendientes a mejorar la calidad académica de los estudiantes de pre-grado. En este contexto, Chile, en los últimos años, y con el objetivo de detectar los problemas de rendimiento académico, ha utilizado un Sistema de Medición de Calidad de la Educación (SIMCE). Esta prueba, realizada en la enseñanza primaria y secundaria, ha dejado en evidencia, entre otras cosas, que la formación (de calidad) del estudiante depende en gran medida de su nivel socioeconómico, existiendo una diferencia notable en la formación que entregan los colegios privados de élite respecto de los colegios públicos. Las investigaciones, en este sentido, han mostrado que la educación chilena está fuertemente segregada desde el punto de vista socioeconómico de tal manera que lo que garantiza una real educación de calidad, es pertenecer a los grupos socioeconómicos altos. Por el contrario, los estudiantes de ingresos económicos bajos y medios, asisten a escuelas y colegios que no logran hacer que sus estudiantes alcancen los aprendizajes mínimos necesarios para enfrentar el rigor de la enseñanza universitaria. Así, ha quedado demostrado en los resultados de la prueba SIMCE que se ha aplicado en los últimos años. Para mayor abundamiento, en lo que respecta a la Universidad de Atacama, en la prueba de selección universitaria un estudiante que obtiene 475 puntos ha respondido 7 preguntas de un total de 75. Un estudiante que obtiene 600 puntos ha respondido alrededor de 30 preguntas de un total de 75. La mayoría de los estudiantes que ingresan a la Universidad de Atacama, han obtenido entre 475 y 600 puntos en dicha prueba. En las últimas décadas la PSU ha puesto de manifiesto las enormes desigualdades educacionales en Chile. Según las cifras del Ministerio de Educación, en la última prueba, el 59 por ciento de los 316 puntajes nacionales provienen de colegios particulares pagados, 29 por ciento de la educación particular subvencionada y 12 por ciento de colegios municipalizados (públicos). Por lo demás, los resultados de la prueba SIMCE son consistentes con estudios internacionales que han revelado que la procedencia del tipo de establecimiento tiene un efecto posterior sobre el rendimiento académico universitario.

Las causas del bajo rendimiento académico deben buscarse más allá del estudiante mismo. Para Fullana (1996), el fracaso estudiantil es un conjunto de factores interrelacionados tanto internos como externos al estudiante. Debido a su naturaleza multifactorial, la literatura económica propone un marco conceptual a partir de una función de producción que incorpora una serie de variables explicativas de dicho desempeño académico. La mayoría de estos estudios se han basado en la función de producción en la que se han incluido variables tales como: la aptitud (cantidad de tiempo que necesita un estudiante para aprender una determinada tarea), oportunidades de aprender (cantidad de tiempo disponible para el aprendizaje), capacidad para aprender la instrucción (habilidades de 
aprendizaje), calidad de la enseñanza (si la calidad de la enseñanza es mala el tiempo necesario para asimilar se incrementará) y la perseverancia (Carroll, 1963). Posteriormente, en un intento por determinar la importancia de las variables socioculturales en el desempeño académico, Coleman (1963) elabora un informe en el cual se sugiere que, teniendo en cuenta los antecedentes familiares y personales de los alumnos, su rendimiento apenas guardaba relación con los recursos escolares. A pesar de las críticas metodológicas que a este trabajo se plantearon (Bowles y Levin, 1968), el informe Coleman dejó abierto un debate que todavía está vigente, en el sentido de que plantea la interrogante si la institución educativa cumple o no un rol determinante o por lo menos importante en el desempeño académico del escolar. Más adelante y con el objetivo de completar el estudio de este autor se trataría de modificar la función de producción anterior para incorporar el tiempo de aprendizaje del estudiante (Levin y Tsang, 1987; Millot, 2002). Estudios posteriores han sugerido que podrían existir otras variables igualmente importantes que tienen que ver con la motivación del estudiante, el auto- concepto académico (conocimiento de sí mismo en el sentido de conocer sus propias posibilidades y limitaciones) y el bienestar psicológico. En general, estos hallazgos han permitido entender mejor los factores asociados al desempeño académico de los estudiantes de manera de diseñar políticas tendientes a mejorar la calidad educativa.

\section{BREVE REVISIÓN DE LA LITERATURA}

En un estudio llevado cabo por Rose et al. (2005) en la Universidad de Western de Australia se analizaron múltiples factores que podrían influir en el rendimiento de los estudiantes. En la investigación se incluyeron variables tales como la habilidad académica del estudiante, el nivel socioeconómico, las características demográficas (lugar de procedencia), tipo de establecimiento educacional, público o privado, y puntaje prueba de acceso a la universidad. En cuanto a las variables puntaje obtenido en la prueba de acceso a la universidad, tipo de colegio (privado o público) y el nivel socioeconómico, se encontró que son estadísticamente significativas, es decir, parecían afectar el rendimiento académico de los estudiantes. Un hallazgo interesante reveló que las calificaciones obtenidas por los estudiantes en la universidad son en gran medida influenciadas por las características individuales del individuo. En la misma línea de investigación, Mills et al. (2009) llevaron a cabo un estudio a un total de 381 alumnos del primer año de la carrera de ciencias de la salud de la Universidad de Western de Australia Occidental, desde el inicio del curso del año 2000 hasta el 2005. El objetivo de la investigación era determinar las variables más relevantes asociados al rendimiento académico. Los autores incluyeron variables tales como la puntuación en el ingreso a la universidad la condición étnica y la asistencia a una escuela pública secundaria. En cuanto a la prueba de acceso a la universidad, los hallazgos fueron consistentes con otros resultados en el sentido de que el rendimiento de los alumnos estaba asociado a una puntuación alta de ingreso a la universidad [Aiteken, 1982; Korn et al., 1987]. El estudio también concluyó que el rendimiento académico de los años posteriores estaba asociado a las notas obtenidas en el primer año. Respecto de la condición étnica se encontró que el desempeño suele ser más bajo en los estudiantes indígenas y que éstos tenían tres veces más probabilidades de dejar sus estudios que los estudiantes no indígenas. Otros trabajos han encontrado 
que los estudiantes con un alto rendimiento académico tienen más probabilidades de permanencia en la universidad respecto de aquellos con bajo rendimiento académico (Bean, 1982; Murtaugh et al., 1999; Houn et al., 2000; Potts et al., 2003; Krause et al., 2005]. En un área de investigación similar, los autores Ibarra et al. (2010) estimaron la probabilidad de mejorar el rendimiento académico de los estudiantes de la Facultad de Ingeniería de la Universidad Nacional de las Naciones, Argentina, para un total de 589 alumnos para el período 1999 - 2003. Para ello, utilizaron la metodología de Regresión Logística. En el estudio incluyeron variables tales como el género (hombre o mujer), nivel de instrucción de los padres, promedio calificaciones enseñanza media y tipo de establecimiento. Luego del análisis, los resultados pusieron de manifiesto que estas variables elevaban, en promedio, dos veces la probabilidad de mejorar el rendimiento académico de los estudiantes.

En cuanto a la variable género como predictor importante sobre el desempeño académico, los autores García et al. (2000) encontraron, para un grupo de alumnos de primer año de la Universidad Carlos III de Madrid para el período 1997-1998, que la variable sexo femenino guardaba una relación estadísticamente positiva con el rendimiento académico. Este hecho podría explicarse sobre la base de que las mujeres asumen un mayor compromiso que los hombres en cuanto a las horas dedicadas al estudio (Rodríguez et al., 2004; García, 2000]. Otros trabajos se han centrado en determinar el efecto que tiene sobre el rendimiento académico las horas que el estudiante dedica al trabajo como actividad complementaria al estudio. Estas investigaciones se han basado en el modelo de suma cero donde el tiempo invertido en el trabajo es tiempo que se extrae al desarrollo de actividades que afectan el desempeño académico (Colleman, 1961; Paul, 1992; Ecktein et al., 1997; Stinebrickner et al., 2003). Sin embargo, otros análisis han encontrado que el trabajo complementario al estudio puede tener un efecto positivo sobre el rendimiento académico [D’Amico, 1984; Schill et al., 1985; Lillydahl, 1990; Turner, 1994). Estos últimos trabajos se fundamentan en la idea Learning by doing que señala que los conocimientos se adquieren con mayor fuerza en la práctica. En este mismo contexto, en un estudio llevado a cabo por Katsikas et al. (2010) para un total de 867 estudiantes de la Universidad de Macedonia, Grecia, para el período académico 1998-1999, se analizó el impacto de la situación del estudiante (trabaja o no) y el origen socioeconómico sobre el desempeño académicos y los años de duración de la carrera. Los autores utilizaron las metodologías de mínimos cuadrados ordinarios y el método de regresión por cuantiles. Contrariamente a lo que se esperaba, los resultados pusieron de manifiesto que los estudiantes que trabajaban no obtuvieron calificaciones más bajas que sus pares que no trabajaban. Se determinó también que la duración de los estudios no estaba relacionada con esta situación y afectaba tanto a los estudiantes que trabajaban como a los que no trabajaban. Otro de los hallazgos es que la prolongación de los estudios parece ser un efecto institucional derivada de las condiciones de escolarización y no de las circunstancias financieras de los estudiantes.

Los estudios de rendimiento académico han sido ampliamente abordados por autores Latinoamericanos. Una característica común de los países Latinoamericanos es la existencia de una clase social que no logra alcanzar los conocimientos básicos en la enseñanza secundaria y que les permita enfrentarse de mejor manera las exigencias de la educación superior. Esta clase presenta características comunes en nuestros países, a saber: deficiencias en el uso del lenguaje y aprendizajes alcanzados en la enseñanza 
media no significativos. En un estudio realizado en la Universidad de Cali por los autores Girón et al. (2005), se encontró que el rendimiento académico de un estudiante viene determinado por los conocimientos previos de lenguaje y matemática, lo que mermaría el rendimiento académico universitario. En este mismo contexto, un estudio realizado por Porto y Digracia (2004) encontró que el nivel de escolaridad de los padres y el desempeño obtenido en secundaria resultaron ser explicativas en el rendimiento universitario. Otros estudios han abordado la problemática del rendimiento académico desde diferentes perspectivas en la que finalmente coinciden en que variables tales como las pruebas aptitud verbal y matemática, el nivel de escolaridad de los padres, número de horas dedicadas al estudios son elementos predictores del rendimiento académico de los estudiantes (Álvarez y García, 1996; González y López, 1985; Porto y Digracia, 2001). En general los hallazgos han permitido entender mejor los factores asociados al desempeño académico de los estudiantes de manera de diseñar políticas tendientes a mejorar la calidad educativa (Seibold, 2000; Garbanzo, 2007).

\section{DATOS Y VARIABLES}

En la elaboración de este trabajo la información fue obtenida a partir de dos fuentes: una encuesta realizada a un total de 258 estudiantes (de un universo de 530 estudiantes) del primer de la Universidad de Atacama para el período académico 2010-2011 y en datos proporcionados por la misma Universidad. Cabe señalar que existe un cierto consenso de lo impreciso que podría resultar medir el rendimiento académico. En este sentido podemos distinguir entre rendimiento inmediato (notas o créditos) y el mediato (logros personales y profesionales. No obstante estas consideraciones las notas (o créditos) parecen ser el mejor indicador o al menos el más accesible para definir el rendimiento académico (Alfaro, 1990; González, 1990; Pérez, 1991).

En nuestro trabajo hemos utilizado como variable dependiente (endógena) el ratio créditos aprobados sobre créditos matriculados para medir el rendimiento académico de los estudiantes. Formalmente, ${ }^{1}$

$$
\text { RENDIMIENTO }(r)=\frac{\text { CREDITOSAPROBADOS }}{\text { CREDITOSMATRICULADOS }} \text { con } 0<r<1
$$

Cuando el ratio es igual a 1 significa que el estudiante ha aprobado todos los créditos matriculados durante el año. En el caso extremo, cuanto el ratio es igual a 0 significa que el estudiante no ha aprobado ninguna de las asignaturas matriculadas. Como variables independientes (exógenas) hemos utilizado la escolaridad de la madre (años de estudio), escolaridad del padre (años de estudio), edad (años). Para capturar el efecto de la prueba de selección universitaria (PSU) hemos utilizado las pruebas verbal y matemática. Como variables de carácter cualitativas (variables Dummy) hemos incorporado, trabaja (1:sí,

1 El rendimiento podría calcularse en forma de porcentaje multiplicando la formula (1) por 100. En tal caso cuando el ratio es igual a 100 el estudiante ha aprobado todos los créditos matriculados. Cuando el ratio es igual a 0 ha reprobado todos créditos matriculados. 
0:no), género (1:hombre: 0:mujer), si el estudiante está conforme con su carrera ( 1:sí, 0:no) y tipo de establecimiento, (1:público 0:particular subvencionado). En la Tabla 1 observamos la descripción de cada una de las variables.

\subsection{PRUEBA DE SELECCIÓN UNIVERSITARIA (PSU) EN CHILE}

La prueba de selección universitaria en Chile se calcula a partir de una serie de factores, esto es: promedio de las notas de enseñanza media (NEM) y pruebas tales como lenguaje y comunicación, matemáticas, historia, ciencias naturales y ciencias. Cada uno de estos factores tiene distinta ponderación (las pruebas de matemática y lenguaje tienen un mayor peso) a la hora de calcular el puntaje de acceso a la universidad. Debido a la importancia de la matemática en el desarrollo del pensamiento lógico, el orden y la rigurosidad y la importancia del leguaje que permiten interpretar, razonar textos y oraciones, hemos creído conveniente aislar el efecto de estas dos tipos de pruebas del resto de los factores que componen la PSU. Esto nos permitirá capturar el verdadero efecto de estas variables sobre el desempeño académico del estudiante. En la Tabla 1 observamos las características de las variables en estudio.

Tabla 1. Descripción de las variables

\begin{tabular}{|c|c|}
\hline Variable independiente & Descripción \\
\hline \multicolumn{2}{|l|}{ Factor relativo al estudiante } \\
\hline Sexo & Mujer, Hombre \\
\hline Edad & $\begin{array}{l}\text { Esta variable se identifica con el grado de } \\
\text { madurez del estudiante. }\end{array}$ \\
\hline Prueba de aptitud matemática & $\begin{array}{l}\text { La prueba de matemática es parte de la } \\
\text { prueba de selección universitaria como } \\
\text { requisito de ingreso a la educación superior. } \\
\text { Los estudios sugieren que podría ser una } \\
\text { variable importante a la hora de analizar el } \\
\text { rendimiento académico del estudiante. }\end{array}$ \\
\hline Prueba verbal & $\begin{array}{c}\text { La prueba verbal es un parte de la prueba } \\
\text { de selección universitaria. Se cree que } \\
\text { podría ser una variable igualmente } \\
\text { importante a la hora de predecir el } \\
\text { desempeño académico. }\end{array}$ \\
\hline Trabaja y Estudia (V. Dummy) & $\begin{array}{l}\text { Variable dicotómica que intenta } \\
\text { capturar el efecto de los estudiantes que } \\
\text { simultáneamente estudian y trabajan } \\
\text { y que podría tener un efecto sobre su } \\
\text { rendimiento académico. }\end{array}$ \\
\hline Conformidad con la carrera (V. Dummy) & $\begin{array}{l}\text { Variable dicotómica que intenta capturar } \\
\text { el efecto de la motivación del estudiante } \\
\text { sobre el desempeño académico. }\end{array}$ \\
\hline
\end{tabular}




\begin{tabular}{|c|c|}
\hline Determinantes sociales & \\
\hline Escolaridad de la Madre & $\begin{array}{c}\text { Variable que mide los años de estudios de } \\
\text { la madre como posible variable predictora } \\
\text { del desempeño académico universitario. } \\
\text { En Chile 12 años de escolaridad significa } \\
\text { enseñanza media completa. }\end{array}$ \\
\hline Escolaridad de la padre & $\begin{array}{c}\text { Factor que mide los años de estudios } \\
\text { del padre como variable igualmente } \\
\text { importante a la hora de analizar el } \\
\text { desempeño académico universitario. }\end{array}$ \\
\hline Factor asociado a la institución & \\
\hline Tipo de establecimiento (público o \\
privado)
\end{tabular}

Fuente. Elaboración propia.

\section{METODOLOGÍA}

En la etapa inicial del trabajo llevaremos a cabo un análisis descriptivo de cada una de las variables incorporadas en el estudio. El objetivo es poder inferir características del conjunto de datos (población). En este tipo de análisis haremos uso de los estadísticos que son considerados estimadores de los parámetros de una población. Específicamente haremos hincapié en las medidas de tendencia central y medidas de dispersión (desviación estándar). Ellos nos permitirá entender mejor la naturaleza de los datos.

En la segunda parte de la investigación haremos uso de la metodología econométrica de Regresión Múltiple con datos de corte transversal para determinar los factores determinantes del rendimiento académico de los estudiantes de la Universidad de Atacama. Formalmente, el modelo econométrico se expresa de la siguiente forma:

$$
Y_{t}=\beta_{0}+\beta_{1} x_{1}+\beta_{2} x_{2}+\ldots+\beta_{k} x_{k t}+\varepsilon_{t} \text { con } t=1,2,3 \ldots . T
$$

Donde $\beta_{1}, \beta_{2}, \beta_{k}$ denotan la magnitud del efecto que las variables explicativas $(x)$ tienen sobre la variable dependiente $(y)$. El coeficiente $\beta_{0}$ se denomina término constante. El término $\varepsilon_{t}$ se denomina término error del modelo con media cero, $E(\varepsilon)=0$, varianza constante, $\operatorname{Var}(\varepsilon)=\sigma^{2}$, y las perturbaciones no correlacionadas, $\operatorname{Cov}\left(\varepsilon_{t}, \varepsilon_{s}\right)=0$ para todo $\forall t \neq s$.

En la tercera parte de la investigación hemos creído conveniente determinar la dependencia de la probabilidad del estudiante de obtener un buen rendimiento académico en función de las variables explicativas antes mencionadas. Para ello haremos uso del Modelo de elección binaria Regresión Logística. Hemos considerado dos categorías: la primera para quienes han aprobado el 50 por ciento de los créditos (buen rendimiento) y la segunda para quienes aprobaron menos del cincuenta por ciento de los créditos (mal 
rendimiento). Queda de esta manera establecida la variable dependiente $(Y)$ dicótoma, en la cual se asigna el valor " 1 " a la primera categoría y "0" a la segunda y un vector $X$ de dimensión $K$, que comprendería las variables exógenas predictoras del rendimiento académico $Y_{l}$. El modelo de regresión logística utiliza la función de distribución logística para modelizar la probabilidad de $Y_{1}=1$, condicionada a unos determinados valores de $X_{1}$. Si la probabilidad es alta se concluye que el evento tiene elevadas posibilidades de ocurrir, caso contrario, con valores reducidos (cercanos a cero) de probabilidad.

Para un vector $x=\left(x_{1}, x_{2}, \ldots x_{n}\right)$ de variables independientes, la probabilidad de ocurrencia del evento se calcula mediante una probabilidad condicional, mediante la expresión:

$$
p(y=1 / x)=\frac{e^{\beta_{0}+\beta_{i} x}}{1+e^{\beta_{0}+\beta_{i} x}}
$$

Donde $\beta_{0}$ es el término independiente, $\beta_{i} x=\beta_{1} x_{1}+\beta_{2} x_{2}+\ldots+\beta_{n} x_{n}$, combinación lineal de las variables independientes. La ecuación (2) representa la probabilidad de ocurrencia del evento dadas las características para las variables independientes $\left(x_{1}\right.$, $x_{2}, \ldots x_{n}$ ) para cada sujeto de la población la cual se obtiene mediante una expresión que involucra funciones exponenciales de base "e". Se observa que el denominador de la ecuación es siempre mayor que el numerador, de forma que resulta en todos los casos un valor siempre positivo y menor que la unidad. Un coeficiente positivo aumenta la probabilidad de ocurrencia del evento, en tanto que un signo negativo la disminuye. En cuanto a los estadísticos, hemos utilizado el valor Odds Ratio para analizar la razón de posibilidad y el indicador, Pseudo-R2 de McFadden y el valor de predicción para analizar los resultados del modelo de regresión logística.

\section{RESULTADOS}

\subsection{ANÁLISIS DESCRIPTIVO}

De acuerdo a los datos obtenidos en la Cuadro 1, se puede observar que el valor de la variable que hace referencia a la prueba de aptitud matemática, tiene de media 547 puntos de un máximo de 850 (esto significa que el estudiante ha contestado un poco más del 18 por ciento de las preguntas). Solamente un estudiante ingresó a la universidad con un puntaje superior a la media. Este dato es revelador porque nos muestra el bajo puntaje de ingreso de los estudiantes a la Universidad de Atacama. En el caso de la prueba de aptitud verbal el caso es muy similar, en el sentido de que existe un alto número de alumnos que obtuvo un bajo puntaje en la prueba de aptitud verbal, con una media de un poco más de 545 puntos. En cuanto a los años de estudios de la madre se observa que la media es de años 12 años de estudio, lo que corresponde a la suma de ocho años de primaria y cuatro años de secundaria. El comportamiento de los datos refleja el hecho de que las madres de los estudiantes de la Universidad de Atacama tienen escasa formación educativa. Se ha detectado un caso de ocho años de escolaridad, lo que corresponde solamente a educación primaria completa. Igualmente sucede con la escolaridad del padre, es decir, una media de escolaridad de 12 años y con una distribución 
de los datos alrededor de la media, lo que nos indica una escasa formación educativa de los padres de los estudiantes de esta Universidad de Atacama. Estos últimos datos son ciertamente reveladores por cuanto nos están mostrando el bajo nivel de escolaridad de los padres de los estudiantes. La variable edad nos indica que el promedio de ingreso de los estudiantes a la enseñanza superior es 19 años.

Cuadro 1. Descripción de las variables

\begin{tabular}{|c|c|c|c|c|}
\hline $\begin{array}{c}\text { Variable } \\
\text { independiente }\end{array}$ & Media & $\begin{array}{c}\text { Desviación } \\
\text { Estándar }\end{array}$ & Máximo & Mínimo \\
\hline $\begin{array}{c}\text { Factor relativo } \\
\text { al estudiante }\end{array}$ & 19.2 & 1.43 & 25 & 18 \\
\hline Edad & 547.7 & 45.2 & 701 & 424 \\
\hline $\begin{array}{c}\text { Prueba de } \\
\text { matemática }\end{array}$ & 545.3 & 59.7 & 768 & 425 \\
\hline Prueba verbal & 12.6 & 2.73 & 17 & 8 \\
\hline $\begin{array}{c}\text { Determinantes } \\
\text { sociales }\end{array}$ & 13 & 2.81 & 17 & 8 \\
\hline $\begin{array}{c}\text { Escolaridad de } \\
\text { la Madre }\end{array}$ & & & & \\
\hline $\begin{array}{c}\text { Escolaridad de } \\
\text { la padre }\end{array}$ & & & & \\
\hline
\end{tabular}

Fuente. Elaboración propia a partir de E-view. 4.0.

\section{ANÁLISIS MODELO DE REGRESIÓN MÚLTIPLE}

En la segunda parte de la investigación se llevará a cabo un análisis econométrico cuyo objetivo es detectar las variables predictoras del rendimiento académico. Luego de aceptado los supuestos del modelo econométrico y realizado las transformaciones adecuadas aceptamos el modelo del Cuadro 2. Los resultados han puesto de manifiesto que la variable binaria genero resultó ser estadísticamente significativo. El signo negativo del coeficiente, es consistente con estudios anteriores y refleja el hecho de que una estudiante mujer asume un mayor compromiso en los estudios, lo que se ve reflejado en un mejor rendimiento académico. La variable dicotómica trabaja es estadísticamente significativa, es decir, un estudiante que trabaja asume un menor compromiso con los estudios afectado su rendimiento. Esto podría explicarse sobre la base de que un estudiante que trabaja tiene menos disponibilidad de tiempo al estudio respecto de aquel que con dedicación exclusiva. En los resultados también se observó que la variable dummy que relaciona la conformidad (motivación) con la carrera resultó ser un predictor importante a la hora de valorar su efecto sobre el rendimiento académico. Este resultado es consistente con otros estudios que han encontrado que la motivación conduce a un compromiso con tareas académicas, lo cual está relacionado con el logro (Dweck et al., 1983; Decharms, 1984). Los hallazgos también han determinado que la prueba verbal y la prueba matemática tienen un efecto positivo sobre el rendimiento académico del estudiante. Es decir, alumnos con buenos puntajes en ambas pruebas les permitirá obtener un mejor 
rendimiento en la etapa universitaria. Esto se explica sobre la base de que las capacidades que permiten interpretar, razonar textos y oraciones (prueba verbal) y las capacidades de razonamiento matemático (prueba de aptitud matemática) generan un efecto positivo sobre el desempeño académico. Respecto al tipo de establecimiento (particular subvencionado o público), la escolaridad de la madre y del padre se encontró que no son variables predictoras del rendimiento académico (estadísticamente no significativas). En los dos últimos casos, creemos que un incremento adicional en el nivel de escolaridad de los padres no implica, necesariamente, un mayor desempeño académico. Respecto de la edad del estudiante, ésta no parece como factor predecible. Las investigaciones empíricas en este último aspecto no han sido concluyentes (Porto et al., 2001; Naylor, 2004). Por último y en cuanto a la capacidad explicativa del modelo, se puede observar que, aunque no es muy alta (21\%), nuestro interés se centra más bien en los efectos marginales de las variables por lo que creemos que el valor del coeficiente de determinación (R2) está dentro del rango esperable en una regresión cuando se utilizan micro datos.

Cuadro 2. Variable Dependiente: Rendimiento Académico. Estimación por MCO

\begin{tabular}{|c|c|c|}
\hline Variables explicativas & Coeficiente & p-valor \\
\hline Constante & 0.739593 & 0.0150 \\
\hline $\begin{array}{c}\text { Tipo de establecimiento } \\
\text { (1=público, 0=particular } \\
\text { subvencionado) }\end{array}$ & -0.033620 & 0.3868 \\
\hline Genero (1:varon, 0:mujer) & & $0.0002^{*}$ \\
\hline & -0.141242 & $0.0289^{*}$ \\
\hline Trabaja (1:si, 0=no) & -0.098947 & $0.0168^{*}$ \\
\hline Conformidad carrera ( 1:si, 0=no) & 0.134745 & 0.2398 \\
\hline Edad & 0.011659 & 0.5512 \\
\hline Escolaridad Madre & 0.004130 & 0.4215 \\
\hline Escolaridad Padre & 0.005695 & $0.0041^{*}$ \\
\hline Prueba verbal & 0.000891 & $0.0000^{*}$ \\
\hline Prueba Matemática & 0.001530 & 0.000000 \\
\hline R-squared & 0.212441 & \\
\hline Prob(F-statistic) & & \\
\hline
\end{tabular}

Nivel de significación

$* \mathrm{p}<5 \%$

$* * \mathrm{p}<10 \%$

\section{ANÁLISIS MODELO REGRESIÓN LOGÍSTICA}

En el Cuadro 3 podemos observar los valores de los Odd Ratios de la variables en análisis. Para la variable que hace referencia al género, el coeficiente resulta negativo y su Odd Ratio de 0.394 nos indica que los estudiantes varones tienen menos 0.394 veces de probabilidad de conseguir un buen rendimiento académico (aprobar menos del 50 
por ciento de los créditos). En cuanto a la variable estudia y trabaja el valor del Odd Ratio nos está indicando que el estudiante que estudia y trabaja simultáneamente tiene menos 0.599 veces de probabilidades de obtener un buen rendimiento académico. La variable que hace referencia a la situación en la cual el estudiante se siente conforme con su carrera, nos está indicando que éste tiene 1.978 más de probabilidad de mejorar su rendimiento cuando presenta esta característica. En cuanto a la prueba verbal el valor del Odd Ratio nos está indicando que por cada aumento de 100 puntos en este tipo de prueba la probabilidad de mejorar el rendimiento se incrementa en 1.51 veces. Igualmente sucede con la prueba de matemática, es decir, con un aumento en 100 puntos en este tipo de prueba la probabilidad de mejorar el desempeño académico se incrementa en 3.409 veces. Obsérvese que los resultados son consistentes con los obtenidos por el método de regresión múltiple en el sentido de que las variables han resultado ser estadísticamente significativas a la hora de evaluar su efecto sobre el desempeño académico. Observemos también que la predicción del modelo es bastante buena, un $66.1 \%$ y con un valor del Pseudo-R2 de McFadden que no se acerca a la unidad. Se predice mejor los ceros 53\% (aquellos que obtuvieron menos del $50 \%$ de los créditos matriculados) frente a los unos con un 33\% (aquellos que obtuvieron más del 50\% de los créditos matriculados).

Cuadro 3. Variable Dependiente: Rendimiento Académico. Modelo de Regresión Logística

\begin{tabular}{|c|c|c|c|}
\hline Variables explicativas & Coeficiente & p-valor & Odds Ratio \\
\hline \multicolumn{4}{|l|}{$\operatorname{Exp}(B)$} \\
\hline Constante & 3.504207 & $0.002803^{*}$ & \\
\hline $\begin{array}{l}\text { Tipo de establecimiento ( } 1=\text { público, } \\
0=\text { particular subvencionado })\end{array}$ & 0.056503 & 0.8470 & 1.058 \\
\hline \multicolumn{4}{|l|}{ Genero (1:varon, 0:mujer) } \\
\hline & -0.929836 & $0.0012 *$ & 0.394 \\
\hline Trabaja $(1:$ si, $0=$ no $)$ & -0.511934 & $0.07396 * *$ & 0.599 \\
\hline Conformidad carrera ( $1: \mathrm{si}, 0=$ no $)$ & 0.682249 & $0.0872 * *$ & 1.978 \\
\hline Edad & 0.072288 & 0.5180 & 1.07 \\
\hline Escolaridad Madre & -0.037498 & 0.4930 & 0.963 \\
\hline Escolaridad Padre & 0.053900 & 0.3163 & 1.05 \\
\hline Prueba aptitud verbal & 0.004172 & $0.0860 * *$ & 1.51 \\
\hline Prueba Aptitud Matemática & 0.012267 & $0.0005 *$ & 3.409 \\
\hline Pseudo-R2 de McFadden: & 0.1056 & & \\
\hline \multicolumn{4}{|l|}{$* \mathrm{p}<5 \%$} \\
\hline \multicolumn{4}{|l|}{$* * \mathrm{p}<10 \%$} \\
\hline Predicción Modelo & $66.1 \%$ & & \\
\hline $\operatorname{Prob}($ F-statistic) & $3.43 \mathrm{E}-05$ & & \\
\hline
\end{tabular}




\section{CONCLUSIONES}

La literatura sugiere que el rendimiento académico es de naturaleza multifactorial. Esto significa que en el desempeño académico de los estudiantes intervienen múltiples causas, desde variables que tienen que ver con los determinantes personales hasta factores asociados a aspectos sociales. En este contexto, nuestro trabajo ha puesto de manifiesto que la variable escolaridad de los padres resultó no ser estadísticamente significativa a la hora de predecir el rendimiento académico. Aunque en principio podría parecer inconsistente desde el punto de vista de la teoría económica, este resultado podría explicarse sobre la base de los resultados obtenidos en la prueba Second Internacional Adult Literacy Survey (SIALS) realizada en Chile en el año 1998 que dejó en evidencia que sólo el 8 por ciento de los profesionales chilenos con formación superior terminada lograron comprender y resolver problema de aritmética básica. Así, entonces, la escolaridad universitaria parece no garantizar, en este caso, que un profesional universitario genere un entorno intelectual que sugiera un fuerte mejoramiento en el capital cultural familiar que coadyuve al éxito de sus hijos en la Universidad; menos si es un profesional proveniente de estratos medios y bajos. La universidad de Atacama, por ser una universidad regional enclavada en una región dedicada fundamentalmente a la minería, recibe a los hijos de estos profesionales. Una mayor escolaridad de los padres (y carentes de cultura) por sí solo no parece ser una variable que pueda considerarse predictora del rendimiento académico.

En cuanto a las variables prueba verbal y matemática el estudio ha puesto de manifiesto que son predictores importantes a la hora de analizar el rendimiento académico del estudiante. En cuanto a la interpretación de los coeficientes, debe recordarse que estos nos indican en cuantas unidades cambia la variable dependiente cuando la variable independiente cambia en una unidad. La interpretación del coeficiente de la prueba verbal (0.000891), nos está diciendo que si este tipo de prueba aumentara en 100 puntos, el rendimiento académico aumentaría en 8.9 puntos porcentuales el porcentaje de créditos aprobados. Igualmente sucede con la interpretación del coeficiente de la prueba de matemática (0.001530), es decir, con un aumento de la prueba de matemática en 100 puntos el desempeño académico aumentaría en 15.3 puntos porcentuales el porcentaje los créditos aprobados. Cabe señalar que estos resultados son consistentes con otros estudios internacionales que han logrado determinar que la nota de acceso a la universidad guarda una estrecha relación con el rendimiento académico posterior (universitario). Esto se explica sobre la base de que las capacidades que permiten interpretar y razonar textos y las capacidades de razonamiento matemático podrían tener un efecto positivo sobre el desempeño académico del estudiante. En cuanto a esto último se han encontrado enormes carencias en conocimientos y habilidades matemáticas y de lenguaje que tienen los estudiantes chilenos que ingresan a los primeros años de universidad y que determinan en gran medida el rendimiento académico posterior.

Otro de los resultados obtenidos ha revelado que la procedencia del tipo de establecimiento (particular subvencionado o público) resultó no ser estadísticamente significativo, cuestión que podría explicarse debido a que en las regiones, en particular la tercera región de Chile la calidad de los colegios particulares subvencionados no es esencialmente diferente a la calidad de los colegios públicos; a diferencia de los colegios de la capital donde sí hay colegios de élite. En cuanto a la variable dicotómica que mide el efecto de un estudiante satisfecho sobre el desempeño académico, es consistente con estudios 
anteriores, en el sentido que resultó ser una variable predictora a la hora de medir su efecto sobre el rendimiento académico. Esto nos sugiere que un estudiante satisfecho tendrá un mejor rendimiento respecto de aquel que no lo está. El valor de su coeficiente (0.134745) nos está indicando que un estudiante a gusto con su carrera aumentaría su rendimiento en 13.47 puntos porcentuales. La variable que espera capturar el efecto de un estudiante que trabaja sobre el rendimiento académico también es consistente con estudios anteriores en el sentido de que el tiempo dedicado al trabajo merma el tiempo dedicado al estudio. Específicamente el coeficiente (-0.09847) nos está indicando que un estudiante que estudia y que trabaja simultáneamente hace que los créditos aprobados disminuyan en aproximadamente en 10 puntos porcentuales. Respecto al resultado de la variable dicotómica genero, el valor de su coeficiente (-0.141242) nos está indicando que la condición de estudiante varón hace que el rendimiento académico disminuya en un 14.12 puntos porcentuales. Este resultado podría interpretarse diciendo las mujeres adoptan comportamientos más adecuados a las normas universitarias (perseverancia y acatamiento de reglas). Es posible que la mujer tenga un mayor sentido de la responsabilidad. De allí que el rendimiento de las mujeres sea por lo general superior que el de los hombres.

En la segunda parte del estudio econométrico hemos estimado las variables que inciden en la probabilidad de mejorar el rendimiento académico del estudiante. Para ello hemos creído conveniente utilizar el modelo de Regresión Logística. Luego del análisis los resultados han puesto de manifiesto que las variables: prueba de matemática, prueba de verbal, y las variables de carácter cualitativas, género, conformidad con la carrera, y la variable que intenta capturar el efecto de un estudiante que estudia y trabaja simultáneamente, resultaron ser estadísticamente significativas elevando, en promedio, dos veces la probabilidad de mejorar el rendimiento académico.

A la luz de los resultados obtenidos creemos que, si bien es cierto que el rendimiento universitario va asociado a un buen dominio en las aéreas de pruebas verbal y matemática y a otros factores relativos al estudiante, existen también otros aspectos tales como los hábitos de estudios, la asistencia regular a clases y no menos significativo la actitud positiva hacia la universidad que pueden tener un rol igualmente importante a la hora de analizar los factores asociados al desempeño académico. Por último, este estudio nos ha permitido identificar aquellas variables que mejor puedan ser objeto de intervención de parte de las autoridades universitarias en materia, por ejemplo, del uso de estrategias metodológicas que promuevan el mejoramiento del rendimiento académico de nuestros estudiantes.

\section{REFERENCIAS BIBLIOGRÁFICAS}

Álvarez M. y García H. (1996). Factores que predicen el rendimiento universitario. San Juan de Pasto: Universidad de Nariño.

Alfaro, M. (1990). Hacia un modelo causal del rendimiento académico. Madrid. CIDE.

Aiteken, N. (1982). College Student Performance, Satisfaction and Retention. Journal of Higher Education, vol. 53 n.1, 32-50.

Bowles, S. y Levin, H. (1968a). The determinants of scholastic achievement: an appraisal of some recent evidence. The Journal of Human Re-sources, vol. 3,n.1, pp.3-24.

Bean, J. (1982). Student attrition, intentions and confidence: Interaction effects in path model. Research in Higher Education, vol.17, pp.155-319 
Carroll. A. (1963). Model of school learning. Teachers College Record, vol.64, n.7, 23-733.

Coleman, J. (1963). Equality of educational opportunity. Washington, DC: U.S. GPO.

D'Amico, R. (1984). Does employment during High School impair academic progress? Sociology of Education, Vol. 57, 152-64.

Decharms, R. (1984). Motivation Enhancement in Educational Settings. Research on Motivation and Education. Student Motivation, vol.1, 275-313.

Dweck, C y Elliot, E. (1983). Socialization, personality and social development. Achievement motivation. E.M Hetherington ed.

Ecktein, Z. y Wolpin, K. (1997). Youth employment and academic performance in High Scholl. CEPR Discussion paper.

Fullana, J. (1996). La investigación sobre las variables relevantes para la prevención del fracaso escolar. Revista Investigación educativa, vol.14, n.1, pp.63-90.

García, M. y San Segundo, J. (2003). Rendimiento académico en el primer curso académico. X Jornadas de la Asociación de Economía de la Educación.

Garbanzo, G. (2007). Factores asociados al rendimiento académico en estudiantes universitarios, una reflexión desde la calidad de la educación superior pública. Revista Educación vol.31, n.1, 43-63.

García, F. (2000). Factores escolares que determinan el rendimiento universitario. Revista española de pedagogía, vol.169, n.170, 497- 519.

Girón E. y González, E. (2005). Determinantes del rendimiento académico y la deserción estudiantil, en el programa de Economía de la Pontificia Universidad Javeriana de Cali. Econ. Gest. Desarro, vol. 3, 173 - 201.

González, R. (1990). Principales dificultades en el rendimiento académico en el primer año de carreras de ingeniería. La investigación educativa sobre la Universidad. Madrid: CIDE, 261-279.

González, M. y López E. (1985). Factores del rendimiento universitario. Revista Española de Pedagogía, n. 168, 497-519.

Huon, G. y Sankey, M. (2000). The transition to University: Understanding differences in success. Papers presented at the 4th Pacific Rim year in higher education conference, Queensland, Australia.

Ibarra, C. y Michalus, C. (2010). Análisis del rendimiento académico mediante un modelo Logit. Revista Industrial, vol.9 n.2, 47-56.

Katsikas, E y and Panagiotidis, T. (2010). Student Status and Academic Performance: an approach of the quality determinants of university studies in Greece. The European Institute, GreeSE Paper, $n$. 40, 1-45

Korn, W. y Green, K. (1987). Retaining and Satisfying Students. Educational Record, vol. 68, 36-48.

Krause, K., Hartley, R., James, R., y McInnis, C. (2005). The first year experience in Australian Universities: Findings from a decade of National studies. Melbourne: Centre for the Study of Higher Education, University of Melbourne.

Levin, H. y Tsang, M. (1987). The economics of student time. Economics of Education Review, vol. 6, 357-364.

Lillydahl, J. (1990). Academic Achievement and part-time employment of High school students. Journal of Economics Education, vol.21, n.3, 307-316.

Millot, B. y Lane, J. (2002). The efficient use of time in education. Education Economics, vol.10, n. 2, 209-228.

Mills, C., Heyworth, J., Rosenwax, L., Carr, S., y Rosenberg, M.(2009). Factors associated with the academic success of first year Health Science students. Advances in Health Sciences Education, vol.14, 205-217.

Murtaugh, P. A., Nurns, 1. D.,y Schuster, J.(1999). Predicting the retention of university students. Research in Higher Education, vol.40, n.3, 355-371. 
Naylor, R y Smith, J.(2004). Determinants of educational success in Higher education. En G. Johnes y J. Johnes (Editores), International Handbook in the economics of education. UK: E. E. Publishing.

Porto, A. y Di Gresia, L. (2000). Características y rendimiento de estudiantes universitarios. El caso de la Facultad de Ciencias Económicas de la Universidad Nacional de La Plata. Documento de Trabajo, n. 24. Abril.

Porto, A y Di Gresia (2001). Rendimiento de estudiantes universitarios y sus determinantes. Buenos Aires: Asociación Argentina de Economía Política.

Potts, G., Schultz, B., y Foust. (2003). The effect of freshman cohort groups on academic performance and retention. Journal of College Student Retention, vol.5, n.4, 385-395.

Paul, H.(1992). The impact of outside employment on student academic achievement in Macroeconomics principals. Journal of Economics Education, vol.13, 51-56.

Pérez, R.(1991). La universidad nacional de educación a distancia. Aproximación a la evaluación de un modelo innovador. Madrid: CIDE.

Rose, E. y Miller, P. (2005). The Determinants of Students`Tertiary Academic Success. Working Papers.

Rodríguez, S y Fita, S y Torrado, M. (2004). El rendimiento académico en la transición secundariauniversidad. Revista de Educación. Temas actuales de enseñanza, vol. 334, 391-414.

Seibold, J. R. (2000). ¿Equidad en la Educación? Reflexiones sobre un nuevo concepto de calidad educativa que integre valores y equidad educativa. CSIC Revista Iberoamericana de Educación, $n .23,1-12$.

Stinebrickner, T. y Stinebrickner R. (2003). Working during school and academic performance. Journal of Labor economics, vol.21,n.2, 473-491

Schill, W., McCartin, R. y Meyer, K. (1985). Youth employment: its relationship to academic and family variables. Journal of Vocational Behavior, vol.26, 155-163.

Turner, M. (1994). The effects of part-time work on High school students academic achievement. University of Maryland (mimeo). 
